\title{
Composición botánica de mieles de la península de Yucatán, mediante qPCR y análisis de curvas de disociación
}

\author{
Botanical composition of honeys from the Yucatan \\ Peninsula, by qPCR and dissociation curve analysis
}

\author{
Alejandra Vanessa Castillo Cázaresa, Yolanda Beatriz Moguel Ordóñez ${ }^{b}$, Moisés Alberto Cortés \\ Cruzc, Elsa Espinosa Huertaa, Miguel Enrique Arechavaleta Velascod, María Alejandra Mora Avilésa
}

\begin{abstract}
RESUMEN
El método cuantitativo de reacción en cadena de la polimerasa (qPCR), seguido por análisis de curvas de disociación, fue desarrollado para la detección rápida y simultánea de la composición botánica en mieles de la Península de Yucatán, México. Cinco muestras de miel ciclo apícola 2013 y cinco del 2014, se caracterizaron para definir el contenido de cinco especies de plantas de importancia apícola; Viguiera dentata, Gymonopodium floribundum, Piscidia piscipula, Acacia angustissima y Mimosa bahamensis. Siete iniciadores de genes genéricos (Adh1, Hmg2, Brass lip, Plant 1, Plant nest, Act1, y Helli-all) se emplearon para caracterizar las especies vegetales y las muestras de miel. Al finalizar la amplificación se obtuvo una curva de disociación por reacción representando productos específicos de amplificación. Los resultados obtenidos indicaron que el contenido taxonómico en las muestras de miel fue diferencial M-1 (V. dentata), M-3 (M. bahamensis y G. floribundum), M-4 (G. floribundum), M-8 (M. bahamensis) y M-13 (V. dentata y G. floribundum). M-7, M-11 y M-12 no revelaron tener ninguna de las especies analizadas, mientras que M-14 y M-15 presentaron un patrón de amplificación diferente a las especies incluidas en este estudio; concordando con los análisis palinológicos. P. piscipula no mostró ningún patrón de amplificación con ninguno de los iniciadores de este estudio y A. angustissima no fue identificada en ninguna muestra de miel, aun cuando el análisis palinológico reveló presencia de esta especie en M-3 y M-4, posiblemente derivado de la ausencia de similitud con los genes de estudio.
\end{abstract}

PALABRAS CLAVE: PCR cuantitativo, Miel, Curvas de disociación, Marcadores moleculares genéricos, Melisopalinología.

\begin{abstract}
A polymerase chain reaction quantitative method (qPCR) followed by melting curve analysis was used for fast and simultaneous detection of botanical composition of honey samples from Yucatan Peninsula, Mexico. Ten honey samples from 2013 and 2014 production were collected directly from beekeepers and analyzed for Viguiera dentata, Gymnopodium floribundum, Piscidia piscipula, Acacia angustissima and Mimosa bahamensis content. Seven primers from generic genes (Adh1, Hmg2, Brass lip, Plant 1, Plant nest, Act1, and Helli-all) were used to amplify plant species and honey samples DNA. Comparisons of melting curves among plant and honey samples for each primer amplification, revealed a variable taxonomic content $M-1$ (V. dentata), M-3 (M. bahamensis y G. floribundum), M-4 (G. floribundum), M-8 ( $M$. bahamensis) y M-13 (V. dentata y G. floribundum). M-7, M-11 and M-12 did not have evidence of presence for any of the plant species under study, whilst M-14 and M-15 showed a different plant species amplification pattern. These results correlate to melissopalynological analysis for most cases. P. piscipula was not detected in any honey sample; however, according to melissopalynological analysis $A$. angustissima was present in M-3 and M-4 even though it was unable to detect it, possibly due to a low or no similarity with generic genes sequence.
\end{abstract}

KEY WORDS: Quantitative PCR, Honey, Melting curves, Gene molecular markers, Melissopalynology.

\footnotetext{
Recibido el 14 de septiembre de 2015. Aceptado el 27 de noviembre de 2015.

a Instituto Nacional de Investigaciones Forestales, Agrícolas y Pecuarias (INIFAP). Campo experimental Bajío, Km 6.5 Carr. Celaya-San Miguel de Allende, 38110 , S/N Celaya, Gto. México. Tel: 018000882222 Ext. 85246. mora_alejandra@yahoo.mx. Correspondencia al último autor.

b Campo Experimental Mocochá, INIFAP. México.

c Centro Nacional de Recursos Genéticos, INIFAP. México.

d Centro Nacional de Investigación Disciplinaria en Fisiología y Mejoramiento Animal. INIFAP. México.
} 


\section{INTRODUCCIÓN}

México se encuentra entre los cinco primeros países en producción y exportación de miel a nivel mundial. La calidad de la miel se establece a través de sus diferentes propiedades como el sabor, color y aroma(1,2), mismas que son producto de las especies vegetales de la zona y que le confiere su origen botánico. Actualmente existen algunos esfuerzos por comercializar la miel clasificada, siendo la Península de Yucatán la región que más ha avanzado en este rubro(3).

La Península de Yucatán aporta el 30 \% de la producción nacional de miel y aproximadamente el $90 \%$ se destina al mercado internacional $(4,5)$. Estas mieles poseen características fisicoquímicas y sensoriales específicas debido a que provienen de una gran diversidad de especies nativas propias de la región. Hasta la fecha se han registrado aproximadamente 2,400 especies vegetales, de las cuales 600 son melíferas y 30 de ellas consideradas de gran importancia apícola(6,7,8).

A pesar de la diversidad de especies melíferas existentes, cerca del $90 \%$ de la producción anual de miel en la Península de Yucatán, proviene de dos principales flujos de néctar; floración de tajonal (Viguiera dentata) en los meses de diciembre a febrero y floración de t'sit'silche' (Gymnopodium floribundum) durante marzo a mayo. Ambas especies florecen en el período de más baja precipitación pluvial. En el período de junio a noviembre (período de mayor precipitación pluvial) florecen una alta proporción de leguminosas y enredaderas; sin embargo, solamente se cosecha un $8 \%$ del total anual(9).

El método empleado más comúnmente, para distinguir las especies vegetales que dieron origen a una miel, es mediante el análisis palinológico, fundamentado en la morfología particular de cada polen presente en la miel(10-14); sin embargo, existen en la actualidad diferentes metodologías que complementan el análisis melisopalinológico.

\section{INTRODUCTION}

Mexico is among the top five honey producing and exporting countries worldwide. Honey quality is determined based on flavor, color and aroma $(1,2)$, which are the result of the plant species in the zone of production. Plant species of a honey production region provides a specific botanical composition to honey, which is unique to that place of origin. Efforts on marketing classified honey based on botanical origin are increasing, particularly in Yucatan Peninsula region(3). Yucatan Peninsula accounts for $30 \%$ of total production from which, $90 \%$ is for international markets $(4,5)$. Honey has specific physicochemical and sensorial traits that respond to a broad diversity of plant species in each region. Approximately 2,400 plant species have been recorded nationally, 600 of which are visited by honey bees and 30 of them are important in honey production $(6,7,8)$.

Ninety percent of annual honey production in the region depends on two main nectar sources: Viguiera dentata (sunflower goldeneye; local term is tajonal) from December to February; and Gymnopodium floribundum (dzildzilche; local term is t' sit'silche') from March to May. Both species bloom during the driest time of year. During the rainy season, from J une to November, a large variety of legumes and vines flower, but they account for only $8 \%$ of the total annual honey harvest in the region(9).

A widely used method to identify among the plant species from which, a honey has been produced is pollen analysis, based on the specific morphology of the pollen(10-14). However, currently there are a number of different methodologies that can complement melissopalynological analysis.

Pollen content in honey is extremely low (trace levels), however, it varies depending on the floral abundance in each zone, and their nectar and pollen contribution, which results in the under- or over-representation of some species. This highlights the need for additional methods that depend less on pollen traces to estimate 
El contenido de polen en las mieles es muy bajo (niveles trazas) pero varía dependiendo de la riqueza floral de cada zona y del aporte de néctar y polen de cada especie vegetal, por lo cual, algunas especies se encuentran sub- 0 sobre-representadas en el perfil palinológico y se reconoce la necesidad de establecer otras metodologías menos dependientes de las trazas de polen en la miel, como la proteómica(15), la espectroscopia óptica y sensores electrónicos(16) o espectrometría de fluorescencia para estimar parámetros físico-químicos y contenido floral(17), entre otros.

Una opción para identificar las especies vegetales presentes en la miel, son los métodos analíticos basados en el ADN; adecuados para la normalización como base para la armonización en la Unión Europea y otros países(18). En este sentido, la definición del origen botánico de las mieles con fines de proporcionar un valor agregado al producto, establece la necesidad de contar con un sistema de detección de secuencias nucleotídicas homólogas, empleando oligonucleótidos genéricos derivados de genes metabólicos que, si bien son afines a todas las especies, también tienen patrones de amplificación muy particulares entre familias de plantas.

Este estudio se centró en el desarrollo y validación de sistemas de amplificación de oligonucleótidos genéricos por PCR cuantitativo (qPCR) empleando SYBR-Green y análisis de las curvas de disociación, para la caracterización de especies de plantas de importancia apícola en la península de Yucatán presentes en la miel a través del polen, como una herramienta para identificar el origen botánico de las mieles.

\section{MATERIAL Y MÉTODOS}

Muestras de miel. Diez panales con al menos el $80 \%$ de operculado se colectaron del apiario del Campo Experimental Mocochá, Yucatán, ubicado en km 1.5 carretera Mocochá-ex Hacienda Carolina, a 210 07' LN y 89o 26' LO, a $10 \mathrm{msnm}$, durante los diferentes ciclos de honey physicochemical parameters and botanical content, such as proteomics(15), optical spectrophotometry and electronic sensors(16), and fluorescence spectrophotometry(17), among others.

An effective method for identifying the botanical source are those based on DNA, suitable for the honey quality regulations used for standardization within the European Union and other countries(18). Defining honey botanical sources with the aim of adding value to the final product requires detection of homologous nucleotide sequences using generic oligonucleotides derived from metabolic genes. These genes are common to all plant species but have amplification patterns highly specific to each plant family.

The objective of the present study was to develop and validate generic oligonucleotide amplification system using quantitative PCR and SYBR ${ }^{\circledR}$ Green, as well as dissociation curve analysis, in order to characterize the plant species and to identify pollen sources in honey production from the Yucatan Peninsula.

\section{MATERIAL AND METHODS}

Honey samples. Ten honeycombs with at least $80 \%$ operculation were collected from the apiary at the Mocochá Experimental Station $\left(21^{\circ} 07^{\prime} \mathrm{N}, 89^{\circ} 26^{\prime} \mathrm{W} ; 10 \mathrm{~m}\right.$ asl) of the National Institute of Forestry, Agricultural and Livestock Research (Instituto Nacional de Investigaciónes Forestales, Agrícolas y Pecuarias - INIFAP) during different flowering cycles. Honey was extracted from the honeycombs using a stainless steel extractor. The extractor was washed with water and dried with $70 \%$ alcohol to prevent contamination between honey samples. After extraction, each honey sample was filtered and stored at room temperature in polyethylene terephthalate (PET) containers. All samples were sent to the Vegetal Tissue Culture and Genetic Engineering Laboratory at the Bají Experimental Station (INIFAP). Five samples were collected during the 2013 season (M-1, M-3, M-4, M-7 
floración que se presentan en la región. Los panales se llevaron al laboratorio para extraer individualmente la miel utilizando un extractor de acero inoxidable, el cual se lavó y secó con agua y alcohol al $70 \%$ para evitar contaminaciones entre muestras. Las muestras se filtraron y almacenaron a temperatura ambiente en frascos de polietileno tereftalato (PET) para su posterior envío al laboratorio de Cultivo de Tejidos Vegetales e Ingeniería Genética, en el Campo Experimental Bajío del INIFAP. Se obtuvieron cinco muestras de miel (M-1, M-3, M-4, M-7 y M-8) del ciclo apícola 2013 y cinco (M-11, M-12, M-13, M-14 y M-15) del ciclo 2014. Para tener de referencia el origen floral de las mieles, se realizó el análisis palinológico de cada muestra.

Análisis melisopalinológico. Las mieles se acetolizaron de acuerdo al método reportado por Louveaux(19). Los gránulos de polen acetolizados se montaron en portaobjetos con gelatina glicerinada y se observaron sus características estructurales como forma, tamaño, aberturas y elementos estructurales utilizando un microscopio Motic BA 410 provisto de un analizador de imágenes. Los gránulos de polen se clasificaron e identificaron de acuerdo a las referencias publicadas por Palacios et al(20).

Material Vegetal. Se recolectó en los alrededores del apiario del Campo Experimental Mocochá, hojas de cinco especies de importancia apícola; Viguiera dentata (tajonal) (florece diciembre a febrero), Gymonopodium floribundum (t'sit'silche') (florece febrero a abril), Piscidia piscipula (ja' bin) (florece marzo a abril), Acacia angustissima (xáax $\mathrm{x}$ ) (florece junio a agosto) y Mimosa bahamensis (sak káatsim) (florece julio a noviembre), para su análisis molecular y utilizarlo como referencia de cada especie.

Extracción de ADN. La extracción de ADN de plantas se realizó empleando el kit comercial DNeasy Plant Mini (Qiagen ${ }^{\circledR}$ ), siguiendo la metodología descrita por el proveedor. La extracción de ADN de miel se realizó empleando $50 \mathrm{~g}$ de miel por cada muestra distribuyéndose and $\mathrm{M}-8$ ) and five samples during the 2014 season (M-11, M-12, M-13, M-14 and M-15). Palynology analyses were done of each sample to generate floral origin reference data.

Melissopalynological analysis. The honey samples were acetolyzed following Louveaux(19). Acetolyzed pollen grains identified in the honeys were fixed on slides with glycerinated gelatin. Grain structural characteristics (e.g. shape, size, openings and structural elements) were analyzed under microscope (Motic BA 410) with an image analyzer. Based on their characteristics, pollen grains were classified and identified using the published literature(20).

Plant Material. Leaves of five plant species known as the most important in honey production in the region were collected: Viguiera dentata (flowers December to February); Gymnopodium floribundum (flowers February to April); Piscidia piscipula (fishpoison tree; locally known as ja' bin) (flowers from March to April); Acacia angustissima (prairie acacia; locally known as xáax) (flowers from June to August); and Mimosa bahamensis (Bahama mimosa; locally known as sak káatsim) (flowers from July to November).

DNA extraction. Plant DNA extraction was done using a commercial kit (DNeasy Plant Mini; Qiagen ${ }^{\circledR}$ ) following manufacturer instructions. Honey samples DNA was extracted from $50 \mathrm{~g}$ of honey per sample. This amount was distributed evenly among four $50 \mathrm{~mL}$ tubes (NALGEN ${ }^{\circledR}$ ), approximately $30 \mathrm{~mL}$ doubledistilled sterile water added to each, and incubated in water bath at $40{ }^{\circ} \mathrm{C}$ for $15 \mathrm{~min}$. Each tube was then agitated by inversion, and centrifuged at $10,000 \mathrm{xg}$ for $15 \mathrm{~min}$ until a sediment (i.e. pollen traces) settled. Once pollen traces were obtained, the pollen DNA extraction was performed using protocol published by the European Union Federal Office of Consumer Protection and Food Safety (2011), and modified by the Vegetal Tissue Culture and Genetic Engineering Laboratory. DNA concentration ranged from 40 to $200 \mathrm{ng} / \mathrm{L}$, depending on pollen content. 
en 4 tubos de $50 \mathrm{ml}\left(\right.$ NALGEN ${ }^{\circledR}$ ), se agregaron aproximadamente $30 \mathrm{ml}$ de agua doble destilada-estéril, y se incubó en baño María a $40 \stackrel{\circ}{ } \mathrm{C}$ durante $15 \mathrm{~min}$. Posteriormente cada tubo se agitó por inversión y se centrifugó a $10,000 \mathrm{xg}$ por $15 \mathrm{~min}$, hasta obtener un sedimento (trazas de polen). A partir de este punto se siguió el protocolo de extracción de ADN de trazas de polen en miel publicado por la Oficina Federal de Protección al Consumidor y Seguridad Alimentaria de la Unión Europea (2011), con modificación en el laboratorio de Cultivo de Tejidos Vegetales e Ingeniería Genética del Campo Experimental Bajío del INIFAP. La concentración de ADN osciló entre 40 y $200 \mathrm{ng} / \mathrm{L}$, dependiendo del contenido de polen por muestra de miel.

Iniciadores. Se utilizaron siete iniciadores de secuencias consenso publicadas previamente por Laube et al(18) para establecer los patrones de amplificación de especies vegetales, y posteriormente identificar estos patrones de amplificación y sus curvas de disociación en las muestras de miel: 1) secuencia no codificante de DNA cloroplástico tRNA-Leu de cloroplasto trnL (UAA) intrón consenso de 19 especies incluyendo algas, briofitas, pteridofitas, gimnospermas y angiospermas(18,21) (Plant 1 ), 2) secuencia anidada de Plant 1 (Plant nest)(18), 3) Actina de canola y girasol (Act)(18), 4) Profilina de girasol (Helli-all)(18), 5) Lipasa de canola (Brass-lip) $(18), 6)$ Alcohol deshidrogenasa de sorgo, arroz y centeno (Adh1)(18) y 7) 3-hidroxi metilglutaril CoA de algodón, chile, tomate, papa y tabaco $(\mathrm{Hmg} 2)(18)$.

Amplificación qPCR. La amplificación se realizó empleando el sistema SYBR Green a través de la siguiente mezcla de reacción: buffer Fast Master mix SYBR Green (Applied Biosystems No. Cat. 4385612) 1X, iniciadores derivados de los marcadores genéricos ( 150 nM) y ADN (100 ng). Las condiciones de amplificación requeridas para el equipo $A B I$ PRISM 7000 (Applied Biosystems) fueron 1 ciclo a $95^{\circ} \mathrm{C}$ por $20 \mathrm{seg}$ (pre-desnaturalización), seguido de 40 ciclos a $95^{\circ} \mathrm{C}$ durante 3 seg (desnaturalización) y $60{ }^{\circ} \mathrm{C}$
Primers. Seven previously published consensus sequence primers were used to establish plant species amplification patterns(18), and then to identify these amplification patterns and their dissociation curves in the honey samples: 1) non-coding tRNA-Leu chloroplastic DNA sequence of trnL (UAA) intron, consensus of 19 species including algae, bryophytes, pteridophytes, gymnosperms and angiosperms (18,21) (Plant 1); 2) nested Plant 1 sequence (Plant nest)(18); 3) canola and sunflower actin (Act) (18); 4) sunflower profilin (Helli-all)(18); 5) canola lipase (Brass-lip)(18); 6) sorghum, rice and rye alcohol dehydrogenase (Adh1)(18); and 7) cotton, pepper, tomato, potato and tobacco 3-hydroxymethyl glutaryl CoA (Hmg2)(18).

qPCR Amplification. SYBR ${ }^{\circledR}$ Green system amplification was done with the following reaction buffer: 1X Fast Master mix SYBR ${ }^{\circledR}$ Green buffer (Applied Biosystems No. Cat. 4385612); 150 nM generic marker derived primers; and $100 \mathrm{ng}$ DNA. Amplification conditions (equipment: $\mathrm{ABI}$ PRISM 7000, Applied Biosystems) were: 1 cycle at $95^{\circ} \mathrm{C}$ for $20 \mathrm{sec}$ (pre-denaturation); 40 cycles at $95{ }^{\circ} \mathrm{C}$ for $3 \mathrm{sec}$ (denaturation); and 1 cycle at $60^{\circ} \mathrm{C}$ for $30 \mathrm{sec}$ (alignment and extension). After amplification, a dissociation curve (fusion temperature curve $[\mathrm{Tm}]$ ) analysis was run to verify the presence of specific and non-specific products according to the fusion temperature of each reaction $(22,23)$. Each plant and honey DNA sample was amplified in triplicate to establish amplification pattern consistency.

In order to verify homogeneous DNA concentrations TaqMan ${ }^{\circledR} 18 \mathrm{~S}$ (human18s rRNA No. Cat. 4319413E, Applied Biosystems) commercial probe was used as internal control, together with the TaqMan ${ }^{\circledR}$ Universal PCR Master Mix II with UNG (No. Cat. 4440038, Applied Biosystems) mixture. Reaction elements without DNA template were used as a negative control.

\section{RESULTS}

The internal control (18S) amplification curves validated the standard DNA concentration and 
por 30 seg (alineación y extensión). Al finalizar la amplificación se realizó un análisis de las curvas de disociación (curva de temperatura de fusión (Tm)) para verificar la presencia de productos específicos e inespecíficos según la temperatura de fusión que posean $(22,23)$. Cada muestra de ADN de planta y de miel se amplificó por triplicado para establecer la consistencia del patrón de amplificación.

Como control interno para verificar concentraciones homogéneas se utilizó la sonda comercial TaqMan 18s (human18s rRNA No. Cat. 4319413E Applied Biosystems) utilizando la mezcla TaqMan Universal PCR Master Mix II with UNG (No. Cat. 4440038 Applied Biosystems). El control negativo consistió de los elementos de reacción sin ADN molde.

\section{RESULTADOS}

Las curvas de amplificación del control interno (18S) validaron la calidad la concentración de ADN estándar para el desarrollo de las reacciones subsecuentes con los iniciadores de genes metabólicos genéricos. La concentración establecida fue de $50 \mathrm{ng} /$ reacción, presentando amplificación entre los ciclos 17 y 20, teniendo menor diferencia de separación entre cada una de las réplicas, incrementando la confiabilidad de homogeneidad de una reacción a otra (Figura 1).

Amplificación de genes genéricos en especies vegetales melíferas

El iniciador Plant 1 mostró evidencia de amplificación en todas las muestras de ADN de plantas, no así para el control negativo sin ADN molde (Figura 2A). La amplificación con los iniciadores Plant nest también mostró amplificación en todas las especies; sin embargo, se observó un diferencial en los ciclos de amplificación entre las diferentes especies, es decir, la secuencia de iniciadores de Plant nest demostró tener mayor similitud en las especies M. bahamensis, G. floribundum y $A$. angustissima que en P. piscipula y V. dentata (Figura 2B).
Figura 1. Curvas de amplificación qPCR del gen constitutivo 18S (control interno) de ADN de plantas (Piscidia piscipula, Gymnopodium floribundum, Viguiera dentata, Mimosa bahamensis y Acacia angustissima) (Software 7000 SDS v 1.2.3, Applied Biosystems)

Figure 1. qPCR amplification curves for the $18 \mathrm{~S}$ constitutive gene (internal control) of plant DNA (Piscidia piscipula, Gymnopodium floribundum, Viguiera dentata, Mimosa bahamensis and Acacia angustissima) (7000 SDS v 1.2.3 software, Applied Biosystems)

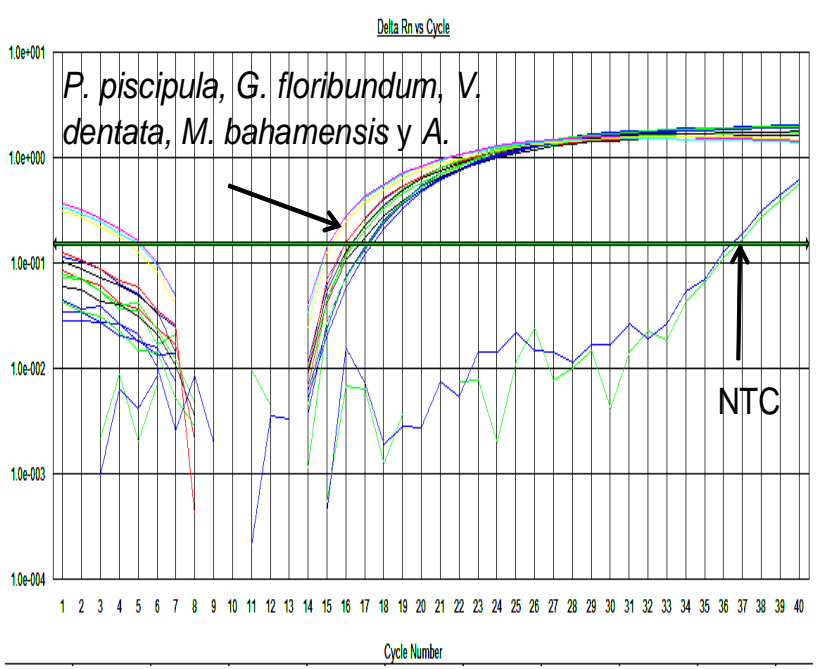

quality for executing the following reactions with the generic metabolic gene primers. The established concentration was $50 \mathrm{ng} /$ reaction, which resulted in amplification between cycles 17 and 20, the least amount of separation between replicates, increasing reliability among reactions (Figure 1 ).

Amplification of generic genes in studied plant species

Primer Plant 1 exhibited amplification in all the plant DNA samples, but not in the negative control (Figure 2A). Amplification of the Plant nest primer was also observed in all five species. However, differences in amplification cycles were present between different species; that is, Plant nest primer exhibited greater similarity with $M$. bahamensis, $G$. floribundum and $A$. angustissima than with P. piscipula and V. dentata (Figure 2B). 
Los iniciadores del gen Actina, Bras-lip y Helliall evidenciaron amplificación en todas las muestras de ADN de plantas en un rango de ciclos diferenciales (Figura 2C, 2D y 2F). Con los iniciadores Hmg2 se observó una amplificación para las muestras de plantas $M$. bahamensis, V. dentata, G. floribundum y A. angustissima, mientras que P. piscipula no
Primers Act, Brass-Lip and Helli-all amplified in all plant DNA samples in a range of different cycles (Figure 2C, 2D AND 2F). Primer Hmg2 amplified in M. bahamensis, $\mathrm{V}$. dentata, G. floribundum and $\mathrm{A}$. angustissima, but did not amplified in P. piscipula (Figura 2E). No amplification was observed with the Adh1 primers in any of the five studied plant species.

Figura 2. Curvas de amplificación $\mathrm{QPCR}$ de $\mathrm{ADN}$ de plantas (V. dentata, G. floribundum, P. piscipula, A. angustissima y M. bahamensis) con el sistema SYBR Green. Iniciadores A) Plant 1; B) Plant nest; C) Actina; D) Brasslip; E) HMG; F) Helli-all. NTC control negativo sin ADN (Software 7000 SDS v 1.2.3, Applied Biosystems)

Figure 2. qPCR amplification curves for the five studied plant species (Piscidia piscipula, Gymnopodium floribundum, Viguiera dentata, Mimosa bahamensis and Acacia angustissima) using the SYBR ${ }^{\circledR}$ Green system. Primers: A) Plant 1; B) Plant nest; C) Actin; D) Brass-lip; E) Hmg2; and F) Helli-all. NTC = negative control without DNA (7000 SDS v 1.2.3 software, Applied Biosystems)
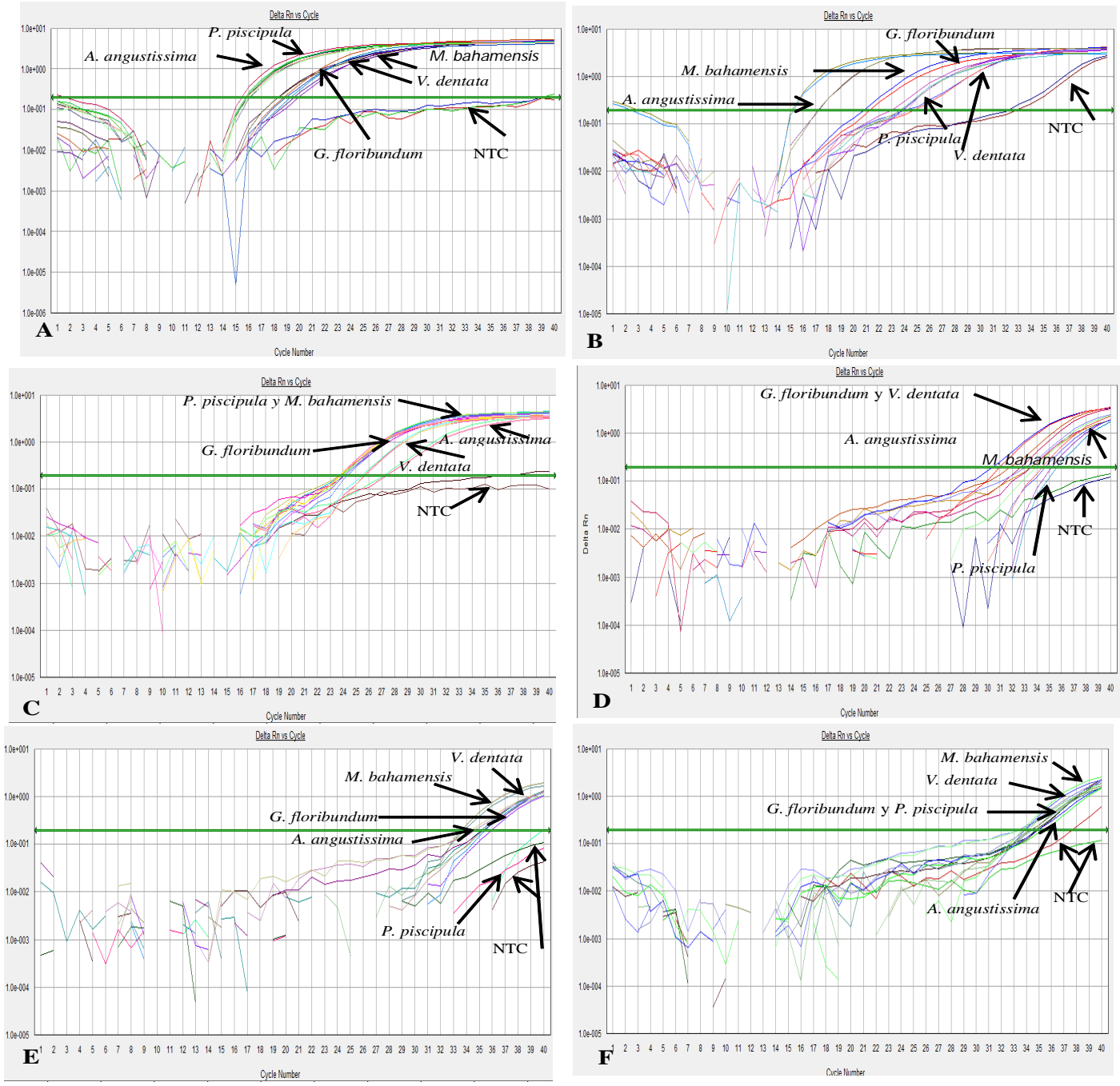
mostró amplificación (Figura 2E). Finalmente, no se observó evidencia de amplificación con los iniciadores Adh1 de alcohol deshidrogenasa en ninguna de las especies de planta bajo estudio.

Análisis de curvas de disociación de los productos de amplificación en especies florales

En las curvas de disociación derivadas de la amplificación con iniciadores Plant 1 se observó un producto específico para cada una de las especies, respecto a la derivada de fluorescencia (el pico máximo de la curva correspondiente a la temperatura de fusión (Tm) del producto) con una variación en las áreas bajo la curva asociado al número de copias del gen en cada una de las especies (Figura 3A). Al igual que Plant 1, cuando se empleó el iniciador Plant nest, la temperatura y el valor de la fluorescencia variaron entre las especies; observándose un producto específico en las curvas de disociación para las cinco especies de plantas (Figura 3C). De esto se confirma que Plant nest reduce el espectro de similitud entre las especies, haciendo más precisa la detección. Sin embargo, es de notar que aun cuando hay amplificación en todas las especies, el control negativo ( $\sin$ ADN molde) también presentó amplificación, lo que establece un fenómeno de amplificación inespecífica derivado posiblemente de formación de dímeros entre los iniciadores eliminando la posibilidad de emplear al iniciador Plant nest.

Los resultados de la amplificación empleando los iniciadores Act del gen actina, muestran que las curvas de disociación muestran un solo producto en todas las especies con variantes en la temperatura y fluorescencia, eliminando así las posibilidades de amplificación cruzada (Figura 3E).

En la curvas de disociación para el iniciador Brass-lip, las especies que obtuvieron un producto específico fueron M. bahamensis y $A$. angustissima (Figura 3G). Las muestras G. floribundum, V. dentata y P. piscipula presentaron dos o más productos inespecíficos,
PCR amplification product and dissociation curve analysis in plant species

A single curve for each species was observed in the analysis of dissociation curves derived from the Plant 1 amplification, when compared to the fluorescence derivative (maximum curve peak corresponded to product fusion temperature - Tm). Variation below the curve was associated with the number of gene copies in each species (Figure 3A). Temperature and fluorescence values also varied between species with the Plant nest primer and single dissociation curves for each of the five plant species were observed (Figure 3C). This confirms that use of Plant nest reduced the spectrum of similarity between species, making detection more accurate. However, even though all five species showed amplification, the negative control amplified as well. This non-specific amplification may have been caused by primer dimer formation, which made Plant nest primer unsuitable for this purpose.

Dissociation curves obtained with Act primer exhibited a single product in all five plant species, with variations in temperature and fluorescence. This eliminates the possibility of crossed amplification (Figure 3E). Brass-lip primer on the other hand, gave single products for $M$. bahamensis and $A$. angustissima (Figure 3G). Curves for G. floribundum, V. dentata and P. piscipula exhibited two or more non-specific curves due to the non-specific binding of the primer to the genome sequences in these three species.

When using Hmg2 primers single curves were observed for $\mathrm{A}$. angustissima and $\mathrm{G}$. floribundum (Figure $3 \mathrm{I}$ ). The fluorescence values produced for Hmg2 gene were relatively lower than the other genes, reducing the size of the area below the curve. This may have been a result of a considerably lower number of $\mathrm{Hmg} 2$ gene copies in the plant species under study, even though there was specificity in the sequences.

Finally, Helli-all primers produced single curves for G. floribundum and V. dentata (Figure 3K). 
Figura 3. Comparación del patrón de curvas de disociación producto de la amplificación con iniciadores de genes genéricos en plantas A: Cloroplasto trnL (UAA) intrón (Plant 1); C: Cloroplasto trnL (UAA) intrón (Plant nest); E: Actina (Act); G: Lipasa (Bras lip); I: 3-hidroxi metilglutaril CoA (Hmg2); K: Profilina (Helli all) y mieles B: Cloroplasto trnL (UAA) intrón (Plant 1); D: Cloroplasto trnL (UAA) intrón (Plant nest); F: Actina (Act); H: Lipasa (Bras lip); J: 3-hidroxi metilglutaril CoA (Hmg2); L: Profilina (Helli all) (Software 7000 SDS v 1.2.3, Applied Biosystems)

Figure 3. Comparison of dissociation curve patterns of amplification products generated with five generic gene plant primers in plant species and honey samples. Plant species: A) Chloroplast trnL (UAA) intron (Plant 1); C) Chloroplast trnL (UAA) intron (Plant nest); E) Actin (Act); G) Lipase (Brass lip); I) 3-hidroxymethyl glutaryl CoA (Hmg2); and K) Profilin (Helli all). Honey samples: B) Chloroplast trnL (UAA) intron (Plant 1); D) Chloroplast trnL (UAA) intron (Plant nest); F) Actin (Act); H) Lipase (Brass lip); J) 3-hidroxymethyl glutaryl CoA (Hmg2); and L) Profilin (Helli all) (7000 SDS v 1.2.3 software, Applied Biosystems)
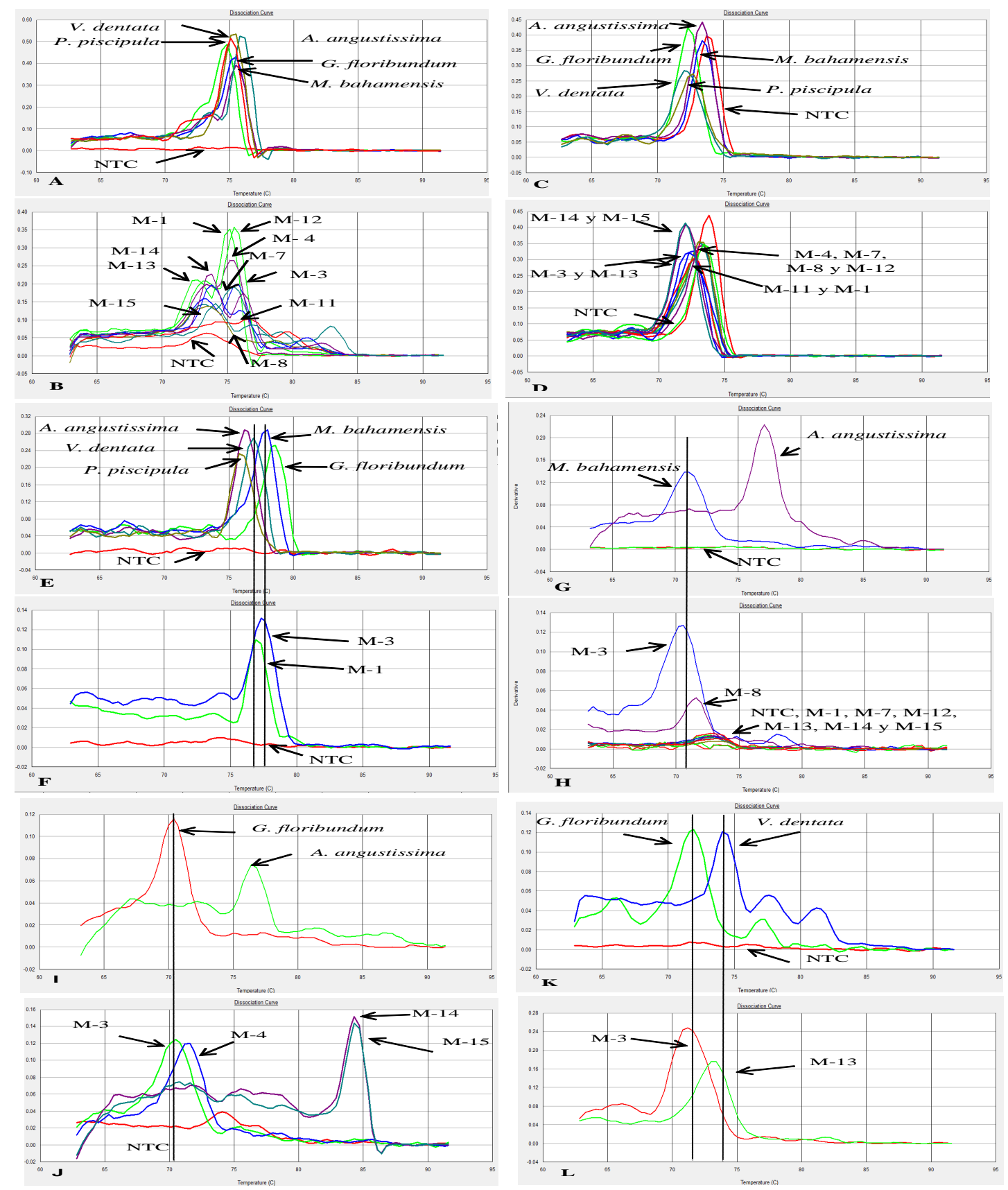
esto derivado de la unión no específica del iniciador a las secuencias en los genomas de estas especies de plantas.

La curva de disociación obtenida en la amplificación con los iniciadores Hmg2 se observó un producto específico para A. angustissima y G. floribundum (Figura 3l). Se observó que con este iniciador los valores de fluorescencia son comparativamente más bajos que con los otros genes, haciendo que el área bajo la curva sea menor, esto puede ser debido a que el número de copias del gen Hmg2 es considerablemente menor en las especies de plantas estudiadas aun cuando hay especificidad en sus secuencias.

Finalmente, en las curvas de disociación obtenidas al amplificar con los iniciadores Helliall, se observó un producto específico para la especie G. floribundum y V. dentata (Figura 3K). Las muestras de M. bahamensis, P. piscipula y A. angustissima presentaron un perfil de desnaturalización diferente (valores de temperatura de fusión distintas) mostrando productos de amplificación inespecíficos.

Análisis de curvas de disociación de los productos de amplificación en $\mathrm{ADN}$ de miel

Una vez realizado el análisis de las curvas de disociación de las especies melíferas, se analizaron y compararon las curvas de disociación derivadas de los productos de amplificación de ADN de muestras de miel (datos no mostrados).

Nuevamente y al igual que ocurrió en la detección en las especies de planta, no se observó evidencia de amplificación con los iniciadores Adhl en ninguna muestra de miel. Una vez más se confirmó que las especies de gramíneas (pastos y cereales), no son parte de la composición taxonómica de las muestras de miel bajo estudio. Con el iniciador Plant 1 del gen cloroplasto trnL (UAA) intrón, se observó amplificación para todas las muestras de ADN de mieles, sin embargo, no se obtuvo un único
Fusion temperature values differed for $M$. bahamensis, P. piscipula and A. angustissima, and non-specific or single curves were recorded for these species.

PCR amplification product and dissociation curve analysis in honey samples

After analyzing the dissociation curves for the studied plant species, the dissociation curves derived from honey samples DNA amplification were analyzed and compared to the plant curves. Similarly to plant species, no amplification was observed with the Adh1 primer in all honey samples. This confirms that graminea species (i.e. grasses and cereals) were not part of the botanical composition of the studied honey samples. All the honey DNA samples exhibited amplification with the Plant 1 primer, although, based on the dissociation curves, no single product was generated in any of the honey samples (Figure 3B).

The same held true for the Plant nest primers where amplification was observed in all honey samples, but no single dissociation curves obtained. Negative control also exhibited amplification, indicating formation of dimers between primers and thus eliminating the possibility of using these primers for accurate detection (Figure 3D).

Act primer produced amplification in all honey samples. In the dissociation curve analysis, sample M-1 exhibited a specific product at 76.9 ${ }^{\circ} \mathrm{C}$ with 0.11 fluorescence, probably due to the presence of V. dentata. Sample M-3 had a specific product at $73.3{ }^{\circ} \mathrm{C}$ with 0.13 fluorescence, in this case probably due to the presence of $\mathrm{M}$. bahamensis (Figure 3E). This was confirmed by the palynological analysis, which identified the presence of $\mathrm{V}$. dentata in $\mathrm{M}-1$ and $\mathrm{M}$. bahamensis in $\mathrm{M}-3$.

Honey samples $M-3, M-4, M-8$ and $M-11$ amplified with Brass-lip primers, however, only $\mathrm{M}-3$ and $\mathrm{M}-8$ had a single curve, both at 70.2 ${ }^{\circ} \mathrm{C}$ and 0.5 fluorescence; indicating the presence of $\mathrm{M}$. bahamensis. This agrees with the 
producto para ninguna muestra de miel de acuerdo al análisis de las curvas de disociación (Figura 3B).

El iniciador Plant nest del gen de cloroplasto trnL (UAA) intrón, también mostró amplificación para todas las mieles, asimismo, este iniciador conservó el patrón de curvas de disociación de la amplificación de ADN de todas las plantas coincidiendo con las mieles; sin embargo, el control negativo (sin ADN molde) también mostró amplificación, evidenciando así la formación de dímeros entre iniciadores y eliminando la posibilidad de hacer una detección confiable con estos iniciadores (Figura 3D).

Cuando se emplearon los iniciadores Act del gen actina se observó amplificación para todas las muestras de miel; sin embargo, en el análisis individual de las curvas de disociación, la muestra de miel M-1 mostró un producto específico a 76.9 ㄷ con una fluorescencia 0.11 , con la probable presencia de la especie $\mathrm{V}$. dentata, mientras que la muestra M-3 obtuvo un producto específico a $73.3 \stackrel{\circ}{\mathrm{C}}$ con una fluorescencia de 0.13 con la oportunidad de presencia de M. bahamensis (Figura 3E). Esto se confirmó con el análisis palinológico, el cual indicó la presencia de $\mathrm{V}$. dentata y $\mathrm{M}$. bahamensis en las muestras $\mathrm{M}-1$ y $\mathrm{M}-3$, respectivamente.

El iniciador Brass lip que amplifica el gen lipasa mostró amplificación para las mieles M-3, M-4, M-8 y M-11; sin embargo, en el análisis de las curvas de disociación las muestras M-3 y M-8 fueron las muestras que presentaron un solo producto específico a 70.2 ㄷ y con una fluorescencia de 0.5. Esto indicó la presencia de $M$. bahamensis coincidiendo en el análisis palinológico encontrando un $45.3 \%$ de gránulos de dicha especie en la muestra (Figura $3 \mathrm{H}$ ). Es de hacer notar que la curva de disociación que acota la amplificación de ADN de A. angustissima no fue obtenida para ninguna de las mieles, no obstante que mediante el análisis palinológico, esta especie sí se encontró en M-3 (22.2 \%) y M-4 (58 \%). palynological analysis, which resulted in $45.3 \%$ $\mathrm{M}$. bahamensis pollen grains presence (Figure $3 \mathrm{H}$ ). The curve that defines amplification of $A$. angustissima DNA was not identified in any of the honey samples, but palynological analysis did identify this species in M-3 (22.2\%) and M-4 (58\%).

Amplification using the Hmg2 primer was observed in all the honey samples except M-1 and $M-8$. The curves indicated that honey samples M-3 and M-4 had a single product with curve characteristics (temperature/fluorescence) similar to the curve from $\mathrm{G}$. floribundum DNA amplification, suggesting that these two samples contained pollen from this species. This agrees with the honey collect date, the flowering period of the species and the palynological analysis (Figure $3 \mathrm{~J}$ ). On the other hand, honey samples M-14 and M-15 showed single curves at a higher temperature and fluorescence value than those observed in M-3 and M-4 with the same Hmg2 primer; the resulting curves for these honey samples did not coincide with any of the curves obtained with the Hmg2 primer for plants under analysis.

The Helli-all primer resulted in amplification of honey samples $M-3, M-4, M-7, M-11, M-12, M-$ 13 and $M-15$. $M-3$ sample showed a single curve that suggested a pattern similar to that of G. floribundum (Figure 3L). This coincides with the result for this sample using the Hmg2 primer, suggesting the presence of this species in this sample (Figure 3J). Sample M-13 exhibited a curve similar to that for $\mathrm{V}$. dentata (Figure $3 \mathrm{~L}$ ).

\section{DISCUSSION}

Characterization of floral origin in honeys produced in Mexico using primers from generic metabolic gene consensus sequences and dissociation curve analysis provides new analytical support for melissopalynology as part of an initial detection stage.

SYBR ${ }^{\circledR}$ Green fluorophore tends to adhere to any amplified product, either the target 
Cuando se empleó el iniciador Hmg2 del gen 3Hidroximetilglutaril CoA se observó amplificación en todas las muestras de miel excepto en M1 y M8. La curva de disociación indica que las muestras $\mathrm{M}-3$ y $\mathrm{M}-4$ presentaron un producto específico similar en características a la curva de disociación (temperatura/ fluorescencia) de la amplificación de ADN de G. floribundum, sugiriendo que estas dos muestras de miel contienen la especie; coincidiendo con la fecha de colecta de la miel, la floración de esta especie y el análisis palinológico (Figura 3J). Es de resaltar que con el iniciador Hmg2, las muestras de miel M-14 y M-15 muestran presencia de un producto específico a una temperatura mayor y a una fluorescencia también superior a la obtenida en las muestras M-3 y $\mathrm{M}-4$; las características de estas curvas de disociación no tienen precedente en la caracterización de ninguna de las especies melíferas analizadas con este iniciador en el presente trabajo.

La amplificación con los iniciadores Helli-all que codifican al gen profilina se obtuvo para las muestras de miel $M-3, M-4, M-7, M-11, M-12$, M-13, M-15. La curva de disociación mostró presencia de un producto específico para la miel M-3 similar al patrón obtenido de la curva de la especie G. floribundum (Figura 3L). Este resultado coincide y ratifica al obtenido con el iniciador Hmg2 donde se sugiere la presencia de esta misma especie (Figuras 3J). Por su parte la muestra de miel M-13 mostró una curva de disociación semejante al patrón de la curva de disociación de la especie V. dentata (Figura 3L).

\section{DISCUSIÓN}

La identificación del origen floral de las mieles producidas en México por medio de la utilización de iniciadores provenientes de secuencias consenso de genes metabólicos genéricos y el análisis con curvas de disociación establecen una nueva posibilidad de apoyo a la melisopalinología en una primera etapa de detección cualitativa. sequence or a non specific DNA sequence, adding up all these signals and producing a single amplification curve. The amplification pattern cannot be used directly to determine specificity, but dissociation curve analysis provides information of a specific amplification. In a dissociation curve, fluorescence decreases gradually as temperature increases(23). By analyzing these dissociation temperature (or fusion) curves, specific products can be identified since curve shape is related to GC (guanine and cytosine) content, and amplicon size and

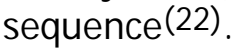

The seven sets of primers from generic gene sequences used here, generated specific amplification patterns even though these sequences are highly conserved in multiple biological families and different kingdoms. The chloroplast trnL (UAA) intron gene, the base for the Plant 1 and Plant nest primers, amplified the DNA samples from all five studied plants. However, this result did not agree with the dissociation curve analysis, which showed nonspecificity with the target sequence in the honey samples DNA. One possible explanation is that since pollen is a masculine germinal cell it has low probabilities of containing plastids (cells from the maternal line), thus limiting identification of DNA chloroplastic sequences from honey samples(24). These results contrast with Laube et al(18), who established that this multicopy sequence found in the chloroplast gene was adequate for detecting low DNA levels in plants and is therefore apt for detecting plant DNA in honey.

Amplification and dissociation curve analysis with Act primer identified $\mathrm{V}$. dentata in honey sample M-1 and $M$. bahamensis in sample M-3. Viguiera dentata blooms between December and January, which coincides with the collect date of sample M-1 (24 January 2013), and the palynological analysis ( $7.5 \% \mathrm{~V}$. dentata). This proportion of pollen in honey sample $\mathrm{M}$ - 1 indicates "minor important pollen" according to the I nternational Apiculture Botanical Commission (Comisión Internacional Botánica Apícola)(19). However, the 
El fluoróforo SYBR ${ }^{\circledR}$ Green suele adherirse a cualquier producto amplificado sea éste el producto blanco o no-blanco, y todas estas señales se suman produciendo una sola curva de amplificación, por lo que el patrón de amplificación no puede ser empleado directamente para determinar especificidad. Para establecer esta especificidad es conveniente realizar un análisis curvas de disociación. En una curva de disociación la fluorescencia decrece de forma gradual a medida que aumenta la temperatura(23). Mediante el análisis de las curvas de la temperatura de disociación (curvas de fusión) es posible identificar productos específicos, cuya forma se relaciona con el contenido de GC (guanina y citosina), tamaño de los amplicones y la secuencia de los mismos(22).

De los siete pares de iniciadores provenientes de secuencias de genes genéricos, fue interesante observar los diferentes patrones de amplificación aun cuando estas secuencias son altamente conservadas en múltiples organismos de familias y reinos divergentes. En un primer análisis se establece que el gen Cloroplasto trnL (UAA) intrón, que conformó la base para el diseño de los iniciadores Plant 1 y Plant nest, amplifican en las muestras de ADN de las cinco especies de plantas bajo estudio; sin embargo, este resultado no concuerda cuando las muestras de miel son sometidas a escrutinio de sus curvas de disociación, al mostrar inespecificidad de la secuencia blanco con las contenidas en las muestras de ADN extraídas de miel. Una posible explicación es que el polen al ser una célula germinal masculina tiene muy bajas probabilidades de contener plastidios (células de herencia materna) limitando así la identificación de secuencias cloroplásticas en ADN proveniente de las muestras de miel(24). Lo anterior contrasta con lo publicado por Laube et al(18), quienes afirman que esta secuencia multicopia en cloroplasto fue adecuada para la detección de bajos niveles de ADN en plantas, siendo aplicable para la detección de ADN de plantas en la miel. fact that the Act primer detected such low levels of pollen content indicates that this primer is highly efficient; being difficult to detect these amounts using traditional methods. Two species were detected in the $\mathrm{M}-3$ sample, $M$. bahamensis (Act and Brass-lip) and G. floribundum (Hmg2 and Helli-all). Nonetheless, it was classified as a M. bahamensis monofloral because this species accounted for $65 \%$ of the pollen grains, making it the predominant pollen in the melissopalynological analysis. Its dominance in this sample coincides with this species' flowering period and the collect date of honey sample. Brass-lip primer confirmed the presence of $M$. bahamensis in $\mathrm{M}-8$ with fusion temperatures similar to those for this species, but the area below the curve was less than that detected for $\mathrm{M}-3$, indicating a lower pollen content.

Both Hmg2 and Helli-all primers confirmed the presence of $\mathrm{G}$. floribundum in honey sample $M-3$. This validates the presence of this species in this honey sample, as well as identifying two generic metabolic genes that produce consistent results, providing additional primers for verification of honey botanical source. Although these genes come from distant plant families (Asteraceae for Helli-all, and Solanaceae for Hmg2), there may be high sequence similarity between the profilin (Helli-all) and 3-hydroxylmethyl glutaryl CoA (Hmg2) genes and their homologues in $\mathrm{G}$. floribundum. For instance, profilin (PRF4) from the Arabidopsis profilins family exhibit greater similarity to the profilins from other species than to the other three Arabidopsis profilins (PRF1, PRF2, PRF3) (25). At the amino acid level, PRF4 was 78.6 to $82.4 \%$ identical to the pollenspecific profilins of monocotyledons such as Zea mays (corn), Phleum pratense (Timothy grass), Betula verrucosa (silver birch), Nicotiana tabacum (tobacco) and Triticum aestivum (wheat).

Hmg2 showed a single dissociation curve in honey samples M-14 and M-15, however, this specific product was not observed in any of the studied plant species; its temperature in the dissociation curves was far higher than those 
Durante el análisis con el iniciador Act del gen actina, se encontró la presencia de $\mathrm{V}$. dentata en la miel $\mathrm{M}-1$ y $\mathrm{M}$. bahamensis en la miel $\mathrm{M}-3$. La especie V. dentata se produce entre el mes de diciembre y enero coincidiendo con la fecha de colecta (24/01/2013) de la muestra M-1 y con el análisis palinológico, mediante el cual se encontró un porcentaje del $7.5 \%$ de la especie. El porcentaje de polen de esta especie encontrado en la miel se considera como "polen minoritario importante" de acuerdo a la Comisión Internacional Botánica Apícola(19); sin embargo, el hecho que el iniciador Act del gen actina lo haya detectado es muy positivo, ya que podría identificarse dicha especie en muy bajos porcentajes difíciles de detectar por el método tradicional. La miel M-3 de la cual se detectaron dos especies M. bahamensis (Act y Brass-lip) y G. floribundum (Hmg2 y Helli-all) se clasificó como monofloral de la especie M. bahamensis, ya que los gránulos se encontraron en un porcentaje del $65 \%$ considerado como "polen predominante" por el análisis melisopalinológico, coincidiendo con la fecha de floración de la especie y la de colecta de la miel. De manera similar, Brass lip confirmó la presencia de M. bahamensis en la muestra M-8 con temperaturas de fusión similares a las obtenidas para la especie; pero con un área bajo la curva inferior a $\mathrm{M}-3$ indicando contenidos bajos de esta especie.

Los iniciadores Hmg2 y Helli-all confirmaron la presencia de $\mathrm{G}$. floribundum en la muestra M-3, corroborando no solo la presencia de la especie en la muestra de miel, sino que además se reportan dos genes metabólicos genéricos que son consistentes en los resultados, proporcionando con esto iniciadores adicionales de verificación. Es interesante notar que si bien estos dos genes provienen de familias distantes (Asteraceae en el caso del iniciador Helli-all y Solanaceae, en el caso de Hmg2), es muy posible que haya una alta similaridad de secuencias entre los genes profilina (Helli-all) y 3-hidroxi metilglutaril CoA (Hmg2) con sus contrapartes homólogas en G. floribundum. En este sentido de acuerdo con Huang et al(25) for the five plant species. These two honey samples were collected at the end of the flowering period of the principal plant species used in honey production. The sequence similarity of these primers are probably associated with secondary species in this cycle such as Mimosa pudica, Acacia gaumeri, Bursera simaruba, Pithecellobium albicans, Senna pallida, Guazuma ulmifolia, Lysiloma latisiliquum and Leucaena leucocephala, among others.

Overall, the most frequent species in the studied honey samples were V. dentata, G. floribundum and $\mathrm{M}$. bahamensis, which bloom for the longest periods among the five analyzed plant species. Acacia angustissima and P. piscipula were not detected in any of the honey samples, most likely because they bloom for only a very short period during the flowering season.

The results indicate that amplification patterns were successfully established for the five studied plant species based on their florescence derivatives. These patterns could then be compared to the patterns detected in honey samples for pollen source identification. Three of the main species present during the season of honey production were identified. Four of the genes (profilin, lipase, 3-hydroxylmethylglutaryl CoA, and actin) contained useful sequences for plant species identification based on amplification patterns which were then validated on honey samples to estimate the presence of one or more plant species.

None of the five plant species were identified in samples M-7, M-11 and M-12, even though the samples were collected during these species' flowering period. This was confirmed by the palynological analysis results in which Asteraceae, Malvaceae and Fabaceae species were identified. Similarly, honey samples M-14 and M-15, which exhibited a distinct amplification pattern possibly related to species not included in the present analysis, showed the presence of Lysiloma bahamensis, Mimosa pudica and Acacia gaumeri after the palynological. 
una de las profilinas que conforman parte de la familia de profilinas de Arabidopsis (PRF4) presentó mayor similitud con otras especies que con las otras tres profilinas de Arabidopsis (PRF1, PRF2, PRF3). A nivel aminoácido PRF4 fue 78.6 a $82.4 \%$ idéntica a profilinas de polenespecífico de monocotiledóneas como Zea mays (maíz), Phleum pratense (hierba timotea), Betula verrucosa (abedul blanco), Nicotiana tabacum (tabaco) y Triticum aestivum (trigo).

La muestras de miel M-14 y M-15 amplificaron el gen 3-hidroxi metil glutaril CoA (Hmg2); sin embargo, se trata de un producto específico que no se observó en la amplificación de ninguna de las especies estudiadas por tener temperatura muy superior a la obtenida para las otras especies en el análisis de las curvas de disociación; además es importante resaltar que estas muestras fueron colectadas al final del periodo de producción de las principales especies florales de importancia apícola, por lo que se considera que la similitud de estos iniciadores está asociada a otras especies secundarias encontradas en el mismo cido, como Mimosa pudica, Acacia gaumeri, Bursera simaruba, Pithecellobium albicans, Senna pallida, Guazuma ulmifolia, Lysiloma latisiliquum y Leucaena leucocephala, entre otras.

En general, las especies que mostraron mayor frecuencia en las muestras de miel fueron V. dentata (tajonal), G. floribundum (t'sit' silche') y $M$. bahamensis (sak káatzim), estas tres especies tienen presencia floral por más tiempo a lo largo del año; mientras que $A$. angustissima (xáax $\mathrm{x}$ ) y P. piscipula (ja'bin) no presentaron evidencia de presencia en ninguna muestra de miel, debido probablemente a que estas especies florecen por periodos muy cortos en la temporada de floración.

De acuerdo con nuestros resultados fue posible establecer el patrón de amplificación de las cinco especies bajo estudio de acuerdo a la derivada de la fluorescencia en cada producto de amplificación, y con ello comparar el patrón obtenido para cada una de las mieles estudiadas.
In the present results, V. dentata was identified by the genes actin and profilin, $M$. bahamensis by the genes actin and lipase, and G. floribundum by the genes Hmg2 and profilin. Acacia angustissima was detected via palynological analysis at very low percentages in honey samples M-4 (6.2 \%), M-8 (2.0\%) and $\mathrm{M}-12(0.5 \%)$. These levels were probably below the detection limit of the markers used in the genetic analysis.

Use of primers other than those used in the present analysis, with a broad similarity in metabolic function, could help to establish amplification patterns for P. piscipula and A. angustissima. In addition, design of new gene amplification primers that do not amplify any of the studied plant species or honey samples (e.g. Adh1) could be part of a strategy to identify other plant species on the Yucatan Peninsula.

\section{CONCLUSIONS AND IMPLICATIONS}

The amplification results as well as the analysis of the dissociation curves for the studied plant species and honey samples, demonstrated that a single gene could be used to detect more than one species (e.g. actin and profilin) in the same sample. Two different genes can also detect the same species in the same sample (e.g. actin and lipase for M. bahamensis, Hmg2 and profilin for $\mathrm{G}$. floribundum), thus confirming and reinforcing detection results. Finally, actin and profilin gene sequences linked to metabolic activity of cell wall function can independently identify the same species (e.g. V. dentata) in honey samples harvested at different times. The detection system presented here, using DNA amplification with generic gene consensus sequences, is an effective protocol that can support melissopalynology analysis in identifying pollen content of specific plant species in honey.

End of english version 
En este sentido se obtuvo evidencia de la presencia de tres de las especies de mayor importancia en el ciclo apícola. De esto observamos que los genes profilina, lipasa, 3-hidroxi metilglutaril CoA y en mayor medida actina, están conformados de secuencias útiles para identificar patrones de amplificación en cada especie y compararlas con las obtenidas en mieles para una aproximación de la posible presencia de una o más especies.

Se encontró que, aun cuando las muestras de miel se cosecharon dentro de los periodos de floración de las cinco especies estudiadas, no se detectó la presencia de dichas especies vegetales en las muestras de miel $M-7, M-11$ y $\mathrm{M}-12$, confirmando dichos resultados con los análisis palinológicos, donde se observaron diferentes especies de Asteraceae, Malvaceae y Fabaceae principalmente. Por otra parte, aún cuando las muestras M-14 y M-15 no indican la presencia de alguna de las cinco especies bajo estudio, demostraron tener un patrón de amplificación diferente y posiblemente relacionado con especies no incluidas en nuestro análisis, concordando con los análisis palinológicos, ya que en dichas muestras de miel se detectaron especies como Lysiloma bahamensis (tzalam), Mimosa pudica (xmuuts') y Acacia gaumeri (box káatsim), entre las mas importantes.

La especie V. dentata fue identificada por los genes actina y proflina, M. bahamensis por los genes actina y lipasa; y $\mathrm{G}$. floribundum por los genes Hmg2 y profilina. Es importante mencionar que la especie $A$. angustissima se detectó por palinología en las mieles $\mathrm{M}-4, \mathrm{M}-8$, y $\mathrm{M}-12$ en muy bajos porcentajes $(6.2,2.0$ y $0.5 \%$ respectivamente) lo cual probablemente estuvo debajo del límite de detección de los marcadores utilizados.

Finalmente, para la caracterización de las especies endémicas que no fueron detectadas por los iniciadores diseñados de secuencias consenso de genes metabólicos generales, se emplearán otros genes con características de amplia similitud en función metabólica a través de especies, que pudieran establecer el patrón de amplificación e identificación de Piscidia piscipula (ja'bin) y Acacia angustissima (xáax $x$ ). Asimismo, el diseño de nuevos iniciadores para la amplificación de genes que no amplificaron para ninguna especie o muestra de miel como el caso de Adh1, podrá establecer una estrategia para la identificación de especies importantes de la Península de Yucatán.

\section{CONCLUSIONES E IMPLICACIONES}

Los resultados de amplificación y posterior comparación de curvas de disociación de plantas y muestras de miel, establecen que un solo gen puede detectar más de una especie (actina y profilina), asimismo, dos genes diferentes en función pueden detectar la misma especie en una misma muestra (actina y lipasa para $\mathrm{M}$. bahamensis y $\mathrm{Hmg}$ y profilina para G. floribundum), confirmando y reforzando con ello los resultados de detección. Por otro lado, secuencias de genes ligados entre si, por su actividad metabólica en pared celular (actina y profilina), identificaron independientemente la misma especie ( $\mathrm{V}$. dentata) en muestras de miel de cosechas diferentes. De esta manera se puede establecer que este sistema basado en detección por amplificación de ADN empleando secuencias consenso de genes genéricos, constituye un nuevo protocolo de apoyo a la melisopalinología para identificar contenido de especies.

\section{LITERATURA CITADA}

1. Bradbear, N. Bees and their role in forest livelihoods. A Guide to the services provided by bees and the sustainable harvesting, processing and marketing of their products. NonWood Forest Products 19, Food and Agriculture Organization of the United Nations (FAO), Rome. 2009.

2. Alfaro BRG, Ortiz J, Viera F, Burgos A, Martínez E, Ramirez E. Caracterización palinológica de las mieles de la Península de Yucatán, Mérida. Universidad Autónoma de Yucatán, Comisión Nacional para el Conociemiento y Uso de la Biodiversidad. 2010.

3. Alfaro BRG, Burgos PAI, Moguel OYB, Godínez GLM, Villanueva GR, Romero RO, et al. Plan rector para promover una denominación de origen de las mieles de la Península 
de Yucatán. Comisión Nacional para el Conocimiento y Uso de la Biodiversidad y Agencia Española de Cooperación Internacional para el Desarrollo 2011.

4. Güemes RF, Villanueva GR. Características de la apicultura en Quintana Roo y del mercado de sus productos. XVI Seminario Americano de Apicultura. Gobierno del Estado de Quintana Roo, Universidad de Quintana Roo. SISIERRA y Ecosur. 2004.

5. SIAP, 2013. Servicios de Información Agroalimentaria y Pesquera. http://www.siap.gob.mx/siaprendes/contenidos/1/ 04-miel/contexto-5.html Consultado Abril 24, 2015.

6. Flores JS. The flowering periods of leguminosae in the Yucatan Peninsula in relation to honey flows. J Apicult Res 1990;29(2): 82-88.

7. Villanueva GR. Nectar sources of European and Africanized honeybee (Apis mellifera) in the Yucatan Peninsula. J Apicult Res 1994;33(1): 44-58.

8. Villanueva GR. Polliniferous plants and foraging strategies of Apis mellifera (Hymenoptera: Apidae) in the Yucatan Peninsula. Rev Biol Trop 2002; 50(3-4): 10.

9. Echazarreta GC, Quezada EJ, Medina ML, Pasteur K. Beekeeping in the Yucatan Peninsula; development and current status. Bee World 1997;73(3):115-127.

10. White JW, Willson RB, Maurizio A, Smith FG. Honey: comprehensive survey. Crane E. editor, William Heineman Ltd. London; 1975.

11. Sawyer R. Honey Identification. Grait Britain: Cardiff Academic Press; 1988.

12. Córdova $\mathrm{CCl}$, Ramírez $\mathrm{AE}$, Martínez $\mathrm{HE}$, Zaldívar CJM. Botanical characterization of honey (Apis mellifera L.) from four regions of the state of Tabasco, Mexico, by means of melisopalynological techniques. Univer Cienc Tróp Húmedo. 2013; 29(1): 163-178.

13. Sarmento STM, Pereira SF, Evangelista RA, Sarmento SEM, Sarmento SG, Santos NJ, et al. Phenolic compounds, melissopalynological, physicochemical analysis and antioxidant activity of jandaíra (Melipona subnitida) honey. J Food Comp Anal 2013;29:10-18.

14. Bobi $O$, Mãrghita LA, Dezmirean DS, Bãrnuiu LI, Mãrgãoan R, Bogdan Gherman B, Bonta V. The Importance of melissopalynology in addition to physical-chemical analysis on botanical authenticity testing of monofloral honey. Bull UASVM Anim Sci Biotechnol 2013; 70(1)24-30.

15. Di Girolamo F, D'Amato A, Righetti PG. Assessment of the floral origin of honey via proteomic tools. J Proteom 2012; 75(12): 3688-3693.

16. Ulloa PA, Guerra R, Cavaco AM, Rosa CAM, Figueira AC, Brigas AF. Determination of the botanical origin of honey by sensor fusion of impedance e-tongue and optical spectroscopy. Comp Electr Agric 2013;94:1-11.

17. Kivima E, Seiman A, Pall R, Sarapuu E, Martverk K, Laos K. Characterization of Estonian honeys by botanical origin. Proceed Estonian Acad Sci 2014;63(2) 183-192.

18. Laube I, Hird H, Brodmann P, Ullman S, Schöne-Michling M, Chisholm J, Broll H. Development of primer and probe sets for the detection of plant species in honey. Food Chem 2010; (118): 979-986.

19. Louveaux J, Maurizio A, Vorwohl G. Methods of melissopalynology. Bee World 1978; (59):1111-1127.

20. Palacio ChR, Ludlow WB, Villanueva GR. Flora palinológica de la reserva de la biósfera de Sian Ka'an, Quintana Roo, México. lera ed. Centro de Investigaciones de Quintana Roo. 1991.

21. Taberlet P, Gielly L, Pautou G, Bouvet J. Universal primers for amplification of three non-coding regions of chloroplast DNA. Plant Mol Biol 1991;17:1105-1109.

22. Ririe KM, Rasmussen RP, Witter CT. Product differentiation by analysis of DNA melting curves during polymerase chain reaction. Anal Biochem 1997; (245): 154-160.

23. Nygren J, Svanvik N, Kubista M. The interactions between the fluorescent dye thiazole orange and DNA. Biopolymers 1998;46(1):39-51.

24. McCauley DE, Sundby AK, Bailey MF, Welch ME. Inheritance of chloroplast DNA is not strictly maternal in Silene vulgaris (Caryophyllaceae): evidence from experimental crosses and natural populations. Am J Bot 2007(94):1333-1337.

25. Huang S, McDowell JM, Weise MJ, Meagher RB. The Arabidopsis Profilin Gene Family. Evidence for an ancient split between constitutive and pollen-specific Profilin genes. Plant Physiol 1999;(111):115-126. 
